\title{
Oxygen uptake and heart rate responses during arm vs combined arm/ electrically stimulated leg exercise in people with paraplegia
}

\author{
Jacqui Raymond ${ }^{1}$, Glen M Davis ${ }^{1}$, Alex Fahey ${ }^{2}$, Mike Climstein ${ }^{1}$ and John R Sutton ${ }^{1}$ \\ ${ }^{1}$ Rehabilitation Research Centre, ${ }^{2}$ Cumberland Health and Research Centre, Faculty of Health Sciences, The \\ University of Sydney, Australia
}

\begin{abstract}
The purpose of this study was to compare the oxygen uptake and heart rate responses during submaximal arm cranking to combined arm cranking + electrical stimulation (ES)-induced leg cycling in individuals with spinal cord injury (SCI). Seven subjects with paraplegia $\left(\mathrm{T}_{4}-\mathrm{T}_{12}\right)$ performed combined arm and leg cycling exercise for $5 \mathrm{~min}$, followed by arm cranking alone at the same power output for a further $5 \mathrm{~min}$. During both exercise conditions, steady state oxygen consumption $\left(\mathrm{VO}_{2}\right)$, carbon dioxide output $\left(\mathrm{VCO}_{2}\right)$, expired ventilation $\left(\mathrm{V}_{\mathrm{E}}\right)$ and heart rate $(\mathrm{HR})$ were determined. The respiratory exchange ratio (RER) and oxygen pulse were calculated from the measured variables. During combined arm + electrical stimulation-induced leg cycling exercise, the $\mathrm{VO}_{2}$ was $25 \%$ higher $\left(1.581 \mathrm{~min}^{-1}\right.$ vs $\left.1.261 \mathrm{~min}^{-1}\right)$, but the $\mathrm{HR}$ was $13 \%$ lower $\left(132 \mathrm{~b} \mathrm{~min}^{-1}\right.$ vs $\left.149 \mathrm{~b} \mathrm{~min}^{-1}\right)$, than during arm cranking exercise alone. Oxygen pulse and $\mathrm{VCO}_{2}$ were also significantly higher (by $42 \%$ and $25 \%$, respectively) during combined arm + ES-induced leg exercise, but there were no differences between the two exercise conditions for $\mathrm{V}_{\mathrm{E}}$ or RER. These data suggest that the absence of the leg 'muscle pump' and a reduced venous return of blood to the heart elevate exercise heart rates during submaximal arm cranking. Conversely, combined arm cranking + ES-induced leg cycling exercise provides the body with a greater metabolic stress than arm cranking alone, while reducing the cardiac stress. The mechanism explaining the heart rate response, however, remains unclear, but may have been influenced by the blood pressure variations across the range of lesions. The findings from this study may have implications for the relative benefit of combined arm + ES-induced leg cycling training for people with paraplegia.
\end{abstract}

Keywords: paraplegia; cardiovascular; electrical stimulation-induced exercise; metabolism

\section{Introduction}

Upper body activities such as wheelchair propulsion or arm cranking, are commonly prescribed modes of exercise for people with spinal cord injuries (SCI). However, for the individual with SCI, the ability to perform these exercises in the seated posture is limited by blood pooling in the dependent lower limbs. ${ }^{1-3}$ The inability to redistribute blood from below the lesion level to the active upper body musculature is a consequence of disruption to sympathetic vascular control and an impaired leg 'muscle pump'.1,4-6

Previous research has reported the effects of disturbed blood redistribution on upper body exercise capacity. ${ }^{6}$ Compared to able-bodied individuals, people with SCI respond to submaximal arm exercise with lower stroke volumes and higher heart rates. ${ }^{2,6-8}$ Furthermore, it has been suggested that submaximal cardiac output may also be lower and blood flow to

Correspondence: Dr GM Davis, Rehabilitation Research Centre, Faculty of Health Sciences, The University of Sydney, PO Box 170, Lidcombe, NSW, Australia, 2141 the exercising arm muscles may be attenuated in response to diminished venous return. ${ }^{1,7,9}$

The use of electrical stimulation (ES) to induce rhythmic contractions of the leg muscles has been shown to lessen venous pooling as observed by elevated stroke volumes and cardiac outputs at rest and during arm exercise. ${ }^{9-11}$ Furthermore, it has been suggested that lower limb ES-induced contractions can improve arm exercise capacity as a result of increased metabolism and augmented hemodynamic performance. ${ }^{12,13}$ In fact, researchers have observed higher oxygen consumptions during maximal arm +ESinduced leg exercise without a change in heart rate when compared to arm exercise alone. ${ }^{13,14}$ Therefore, it is possible that during training with simultaneous arm + ES-induced leg exercise, individuals with SCI can perform at higher levels of metabolic demand versus arm exercise alone.

Although it is generally believed that combined arm + ES-induced leg cycling exercise will elicit a greater metabolic stress during submaximal exercise than arm cranking alone, ${ }^{14,15}$ the heart rate response 
is less clear. To date, only a few studies have directly compared the two modalities of exercise at submaximal levels. Hooker et $a l^{15}$ observed greater oxygen consumptions and higher heart rates during simultaneous arm + ES-induced leg cycling compared to arm exercise alone. In contrast, Raymond et $a l^{14}$ demonstrated elevated oxygen consumptions but no change in heart rates during combined arm + ESinduced leg cycling.

The purpose of the present study was to compare the oxygen uptake and heart rate responses of individuals with paraplegia performing submaximal arm exercise to simultaneous arm +ES-induced leg cycling exercise. In particular, this investigation focused on the oxygen uptake and heart rate adaptations in the transition from arm + ES-induced leg cycling to arm exercise alone. The findings from this study may have implications for the relative value of simultaneous arm + ES-induced leg training for people with paraplegia.

\section{Methods}

\section{Subjects}

Seven subjects with paraplegia $\left(\mathrm{T}_{4}-\mathrm{T}_{12}\right)$ were recruited to participate in this study. The subjects represented a subset of participants in the Shake-a-Leg research project at the Faculty of Health Sciences within the University of Sydney. Prior to participation, all subjects underwent a 12-lead resting ECG and were approved to undertake vigorous exercise by an independent medical examiner. All subjects demonstrated adequate sympathetic control of cardioacceleration based on their heart rate responses to maximal exercise in the range of $162-188 \mathrm{~b} \mathrm{~min}^{-1}$. Additionally, the subjects underwent planar radiographs of their lower limbs and pelvis to ensure adequate bone integrity prior to ES-induced muscle contractions. All subjects were able to leg cycle for at least 5 min at $0 \mathrm{~W}$ resistance on the Ergys 1 leg cycle ergometer (Therapeutic Alliances Inc., Dayton, USA). The subject characteristics are presented in Table 1.

Table 1 Subject characteristics

\begin{tabular}{lcccc}
\hline Subject & Sex & $\begin{array}{c}\text { Age } \\
\text { (years) }\end{array}$ & $\begin{array}{c}\text { Weight } \\
(\mathrm{kg})\end{array}$ & Level of lesion \\
\hline 1 & $\mathrm{M}$ & 36 & 88 & $\mathrm{~T}_{10}$ \\
2 & $\mathrm{~F}$ & 30 & 54 & $\mathrm{~T}_{4}$ \\
3 & $\mathrm{M}$ & 44 & 82 & $\mathrm{~T}_{5-6}$ \\
4 & $\mathrm{~F}$ & 22 & 53 & $\mathrm{~T}_{10-12}$ \\
5 & $\mathrm{M}$ & 28 & 62 & $\mathrm{~T}_{9}$ \\
6 & $\mathrm{M}$ & 28 & 72 & $\mathrm{~T}_{4-6}$ \\
7 & $\mathrm{M}$ & 35 & 60 & $\mathrm{~T}_{5}$ \\
Mean \pm s.e. & & $31.9 \pm 2.7$ & $67.3 \pm 5.2$ & \\
\hline
\end{tabular}

\section{Protocol}

Prior to testing, gel-backed, carbon-rubber surface electrodes (Medtronic, San Diego, USA) were placed over the motor points of the gluteal, quadricep and hamstring muscle groups. Three disposable silver/silver chloride electrodes were also applied in a standard $\mathrm{CM}_{5}$ configuration $^{16}$ for ECG purposes. The subject then transferred on to the Ergys 1 leg cycle ergometer. An arm crank ergometer (Monark, Model 881E, Varberg, Sweden) was mounted on a customized steel frame over the bike to enable simultaneous arm cranking and leg cycling.

The subjects rested for $5 \mathrm{~min}$ in the upright, seated posture. After this pre-exercise habituation period, an assistant turned the foot pedals for $1 \mathrm{~min}$ to ensure there were no spastic contractions in the lower limbs which might interfere with the subsequent leg exercise. The subject was then instructed to undertake arm cranking at a cadence of 50 revs $\min ^{-1}$. At the same time, the legs commenced cycling induced by computer controlled electrical stimulation. The arm crank resistance was set at a level which represented approximately $65 \%$ of the subject's arm $\mathrm{VO}_{2} \max$ as determined prior to testing. The leg cycling ergometer resistance was set at the highest level which the subject could perform for $5 \mathrm{~min}$ (also determined prior to testing). At the end of $5 \mathrm{~min}$ of combined arm cranking + ES-induced leg cycling, the leg exercise was terminated and the subject then continued arm cranking at the same power output for a further 5 min.

\section{Physiological measurements}

During rest and exercise, metabolic and respiratory parameters were determined via open circuit spirometry. Subjects breathed through a two-way valve and expired gas was collected breath-by-breath using a Sensormedics 2900 Metabolic Cart (Sensormedics, Loma Linda, USA). Values for oxygen consumption $\left(\mathrm{VO}_{2}\right)$, carbon dioxide output $\left(\mathrm{VCO}_{2}\right)$ and expired ventilation ( $\mathrm{V}_{\mathrm{E}}$ BTPS) were smoothed with a rolling three breath average. All metabolic and respiratory parameters were then averaged over the 3 rd to 4 th min at rest and during the last minute of arm exercise or combined arm and ES-induced leg cycling exercise. Heart rate was determined by averaging the R-R intervals of the ECG at rest and during exercise over the same time periods used for the metabolic data. The respiratory exchange ratio $\left(\mathrm{VCO}_{2} / \mathrm{VO}_{2}\right)$ and oxygen pulse $\left(\mathrm{VO}_{2} / \mathrm{HR}\right)$, were derived from the measured variables.

Arm cranking and leg cycling power outputs were determined by counting the cadence for $1 \mathrm{~min}$ during combined arm + ES-induced leg cycling exercise and during arm cranking exercise alone. The power output was calculated by multiplying the cadence by the resistance. The total power output was calculated by adding the arm power output and the leg power output. 


\section{Statistics}

The statistical analysis consisted of a repeated measures ANOVA with a posteriori paired $t$-tests to contrast the changes of physiological response magnitudes at rest, during combined arm and ES-induced leg cycling exercise and arm cranking exercise alone. Statistical significance was set at the $95 \%$ confidence limit $(P<0.05)$. All data are presented as means \pm standard error (SE). All statistics were performed using the SPSS for Windows (Version 7.0) statistical package.

\section{Results}

The difference in total power output between arm cranking and combined arm + ES-induced leg cycling $(29.6 \pm 6.3 \mathrm{~W}$ vs $34.9 \pm 7.4 \mathrm{~W}$, respectively) proved to be statistically significant. Three of the seven subjects leg cycled against an unloaded flywheel. Therefore, for these three subjects, the total power output was no different than the arm cranking power output.

The steady state $\mathrm{VO}_{2}$ was $0.25 \pm 0.021 \mathrm{~min}^{-1}$ at rest. During exercise, the steady state $\mathrm{VO}_{2}$ was significantly higher during combined arm + ES-induced leg cycling exercise than during arm cranking exercise alone $\left(1.58 \pm 0.121 \mathrm{~min}^{-1}\right.$ vs $1.26 \pm$ $0.131 \mathrm{~min}^{-1}$, respectively; $\left.P<0.05\right)$. The average $\mathrm{VO}_{2}$ at rest and during the two exercise conditions is presented in Figure 1.

The heart rate at rest was $80 \pm 5 \mathrm{~b} \mathrm{~min}^{-1}$. During the two exercise conditions, the heart rate was significantly increased above resting levels. Heart rate was also significantly higher during arm cranking exercise than during combined arm + ES-induced leg cycling exercise $\left(149 \pm 6 \mathrm{~b} \mathrm{~min}^{-1}\right.$ vs $132 \pm 5 \mathrm{~b} \mathrm{~min}^{-1}$, respectively; $P<0.05)$. There were no differences in heart rate responses between higher lesion level subjects $\left(\mathrm{T}_{4}-\mathrm{T}_{6}\right)$ vs lower lesion $\left(\mathrm{T}_{9}-\mathrm{T}_{12}\right)$ individuals. The average heart rate at rest and during both exercise conditions is presented in Figure 2. The kinetics of the transition in heart rate and oxygen consumption from combined arm cranking + leg cycling to arm cranking exercise are presented in Figure 3.

The steady state expired ventilation was higher during exercise than at rest (Rest: 7.9 \pm 0.71 $\min ^{-1}$; Arm: $43.8+3.91 \mathrm{~min}^{-1}$; Arm + Leg: $44.7 \pm$ $\left.2.2 \mathrm{~min}^{-1}\right)$. There were no significant differences in ventilation between steady state arm cranking and combined arm + ES-induced leg cycling. The average ventilation at rest and during exercise is presented in Figure 4.

The oxygen pulse, $\mathrm{VCO}_{2}$ and respiratory exchange ratio (RER) were significantly increased above resting values during arm exercise and combined arm + ES-induced leg exercise. Oxygen pulse and $\mathrm{VCO}_{2}$ were also significantly higher during combined arm + ES-induced leg cycling than during arm cranking $\left(\mathrm{O}_{2}\right.$ pulse: $12.2 \pm 1.2 \mathrm{ml} \mathrm{bt}^{-1}$ vs $8.6 \pm$ $1.2 \mathrm{ml} \mathrm{bt}^{-1}$, respectively; $\mathrm{VCO}_{2}: 1.56 \pm 0.0811 \mathrm{~min}^{-1}$ vs $1.24 \pm 0.101 \mathrm{~min}^{-1}$, respectively). There was no difference in RER between arm cranking exercise and combined arm + ES-induced leg cycling exercise.

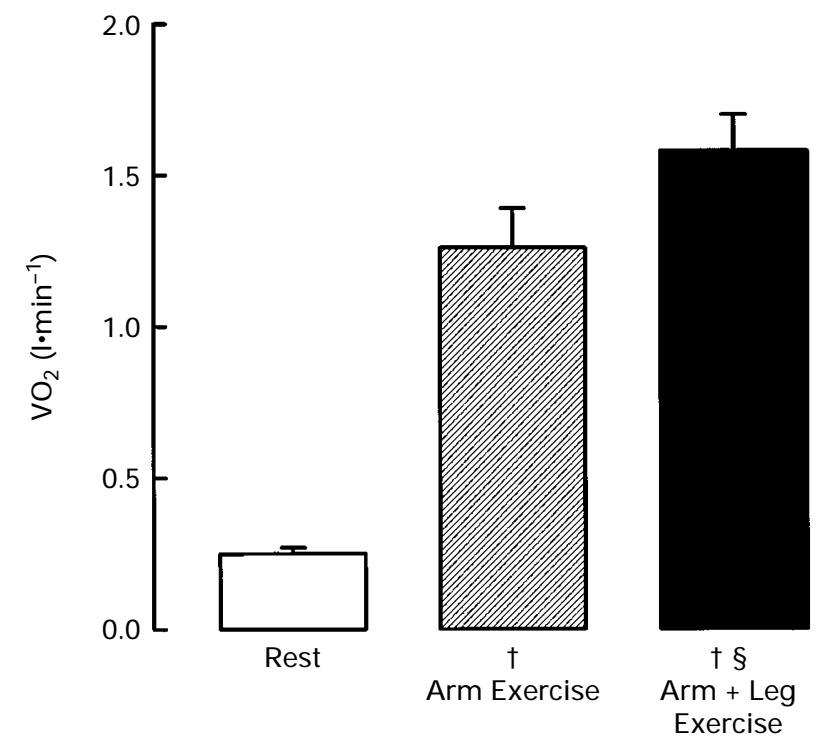

Figure 1 Steady state oxygen consumption at rest, during arm cranking exercise and combined arm cranking + ESinduced leg cycling exercise. Data are mean $\pm \mathrm{SE} . \dagger P<0.05$ compared to rest. $\S P<0.05$ compared to arm cranking exercise

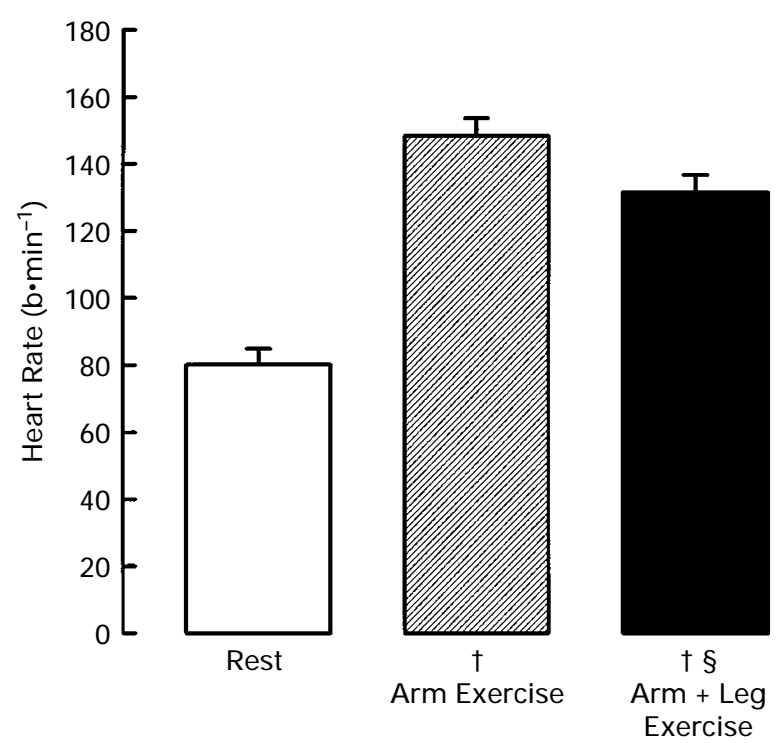

Figure 2 Steady state heart rate at rest, during arm cranking exercise and combined arm cranking + ES-induced leg cycling exercise. Data are mean $\pm \mathrm{SE}$. $\dagger P<0.05$ compared to rest. $\S P<0.05$ compared to arm cranking exercise 


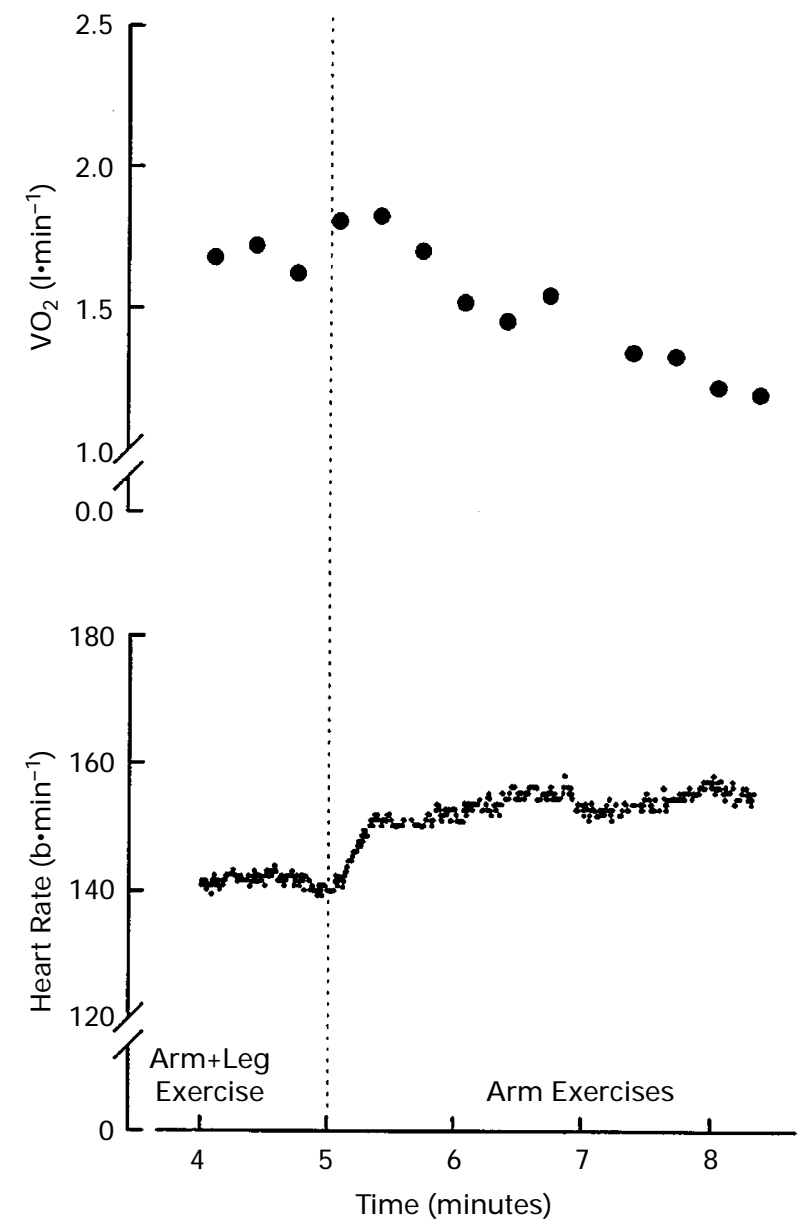

Figure 3 Representation of the kinetics of heart rate and oxygen consumption during the transition from combined arm + ES-induced leg cycling exercise to arm cranking exercise alone. Data were derived from one subject

\section{Discussion}

This study demonstrated that, in people with paraplegia, combined arm cranking + ES-induced leg cycling provides the body with a greater total metabolic stress than arm cranking alone. On the other hand, the cardiac stress is less during combined arm + leg cycling than during arm cranking exercise. These physiological responses were suggested by; (i) the greater oxygen consumption during combined arm cranking + ES-induced leg cycling than during arm cranking, and (ii) the higher heart rate observed during arm cranking compared to combined arm + leg exercise.

The $25 \%$ higher oxygen consumption observed during combined arm + ES-induced leg cycling exercise was probably due to the use of a collectively larger muscle mass. Similar results have been observed in previous studies which have compared arm cranking exercise to combined arm + ES-induced leg cycling

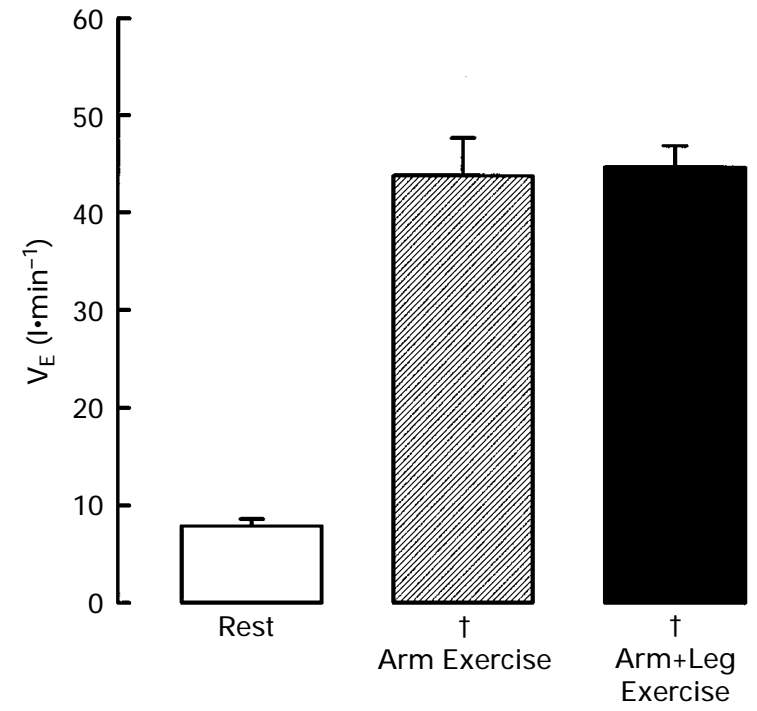

Figure 4 Steady state ventilation at rest, during arm cranking exercise and combined arm cranking + ES-induced leg cycling exercise. Data are mean $\pm \mathrm{SE}$. $\dagger P<0.05$ compared to rest

exercise. ${ }^{14,15}$ It is likely that the greater need for oxygen was due to a combination of 'central' and 'peripheral' factors. Cardiac output may have been increased in response to a greater need for oxygen at the muscle level, but peripheral oxygen extraction may also have been elevated due to the activation of a greater volume of muscle mass. Hooker and colleagues, ${ }^{15}$ in a study of subjects with cervical lesions, noted that the higher $\mathrm{VO}_{2}$ observed during arm + leg exercise compared to arm exercise alone was attributed to a $47 \%$ increase in cardiac output and an insignificant increase (only $5 \%$ ) in a- $\mathrm{vO}_{2}$ difference. Unfortunately, in the current study, it was impossible to determine the exact contribution of each factor.

Whereas the oxygen consumption was lower during arm cranking than during combined arm + leg cycling, the heart rate was $13 \%$ higher. The higher rate observed during arm cranking alone (Figure 2) was elicited within the first minute following the cessation of ES-induced leg cycling (see Figure 3). This transition demonstrated the rapid physiological adjustments which occurred following the cessation of leg exercise. The transfer from arm + leg exercise to arm exercise alone was probably coincident with a fall of venous return resulting from an inactive leg 'muscle pump'. Reduced venous return elicits lower cardiac filling pressures and attenuated stroke volumes. ${ }^{17}$ Therefore it is possible that the increase in heart rate was elicited by the baroreceptors in response to a reduction in stroke volume and probable fall in blood pressure. Unfortunately, in this study, blood pressure was not measured due to the technical difficulties of obtaining accurate readings during arm exercise. 
Therefore this explanation encompassing the role of the baroreceptors must be viewed with caution.

The difference in heart rate we observed between the two exercise conditions is in contrast to the findings of two previous studies. ${ }^{14,15}$ Hooker et $a l^{15}$ noted that exercise heart rate in people with quadriplegia was significantly higher during combined arm cranking + ES-induced leg cycling than during arm cranking alone. A different subject population used in the present study may partly explain the contrasting heart rate responses. The sympathetic control of cardioacceleration is completely impaired in people with tetraplegia, but it is usually not attenuated in those with paraplegia. Therefore, the significant increase in heart rate observed by Hooker et $a l^{15}$ was likely due to predominant parasympathetic withdrawal. In contrast, the paraplegics in the current study could mediate their cardioacceleration not only by parasympathetic withdrawal, but also by sympathetic activation of the heart.

Interestingly, in contrast to the present study and to the findings of Hooker et al, ${ }^{15}$ Raymond and colleagues $^{14}$ have previously observed similar heart rates during arm cranking as during combined arm cranking + ES-induced leg cycling. Differences of research design may explain the seeming disparity of heart rate responses between our earlier study and the current one. In the previous study of Raymond et $a l,{ }^{14}$ subjects with SCI were tested on different days (ie arm exercise on one day, and combined arm cranking + ESinduced leg cycling on the other day). However, in the current investigation, arm + leg exercise always preceded solitary arm effort and this may in part explain the increase in heart rate upon transition to arm cranking exercise. Peripheral vasodilation is a requirement for improved oxygen delivery to exercising skeletal muscle. ${ }^{17}$ In the current study, we propose that ES-induced leg cycling (in combination with arm exercise) induced a peripheral vasodilation in the legs to a greater degree than if the legs were at rest. Therefore, sequencing arm cranking to follow arm + leg exercise may have induced maximal leg muscle vasodilation and venous pooling, perhaps even more than under resting conditions. Reduced venous return upon transition to arm cranking may have compromised stroke volume, thereby eliciting an increase of heart rate to a greater extent than if the legs had not cycled prior to arm exercise.

It is possible that the lower heart rate observed during combined arm cranking + ES-induced leg cycling was due to a higher stroke volume. Oxygen pulse, an indirect indicator of stroke volume, was $40 \%$ higher during combined arm cranking + leg cycling than arm cranking alone. This difference demonstrates that rhythmic muscle contractions elicited via electrical stimulation reactivate the leg 'muscle pump' to enhance venous return. According to the Frank Starling relationship, the increase in venous return augments cardiac preload, effecting a greater stretch of myocardial fibres, to produce a larger stroke volume.
Other factors (eg cardiac contractility, compliance, afterload, autonomic reflexes, etc) also alter stroke volume, but in the current experiment these were probably secondary to enhanced ventricular filling. Increases in stroke volume during combined arm cranking + ES-induced leg cycling have been observed previously in people with tetraplegia. ${ }^{15}$

A significant shortcoming of this study, which limits the interpretation of the results, is the lack of blood pressure measurements. Although sub group analysis of the higher lesion subjects $\left(\mathrm{T}_{4}-\mathrm{T}_{6}\right)$ versus the lower lesion subjects $\left(\mathrm{T}_{9}-\mathrm{T}_{12}\right)$ revealed no differences of the heart rate behaviour, there may well have been different mechanisms explaining the responses across the range of lesions, based on blood pressure changes. For example, the heart rate response to ES-induced leg exercise may have been influenced by the presence of autonomic dysreflexia, a condition sometimes experienced by those with lesions above $\mathrm{T}_{6}$ and associated with a reflex increase in blood pressure. ${ }^{18}$ Or perhaps the heart rate was influenced by exercise-induced hypotension, a condition which has been shown to exist in people with autonomic failure. ${ }^{19}$ However, because the present study did not assess blood pressure changes during either combined arm and ES-induced leg cycling or arm cranking alone, we can only speculate on the possible mechanisms explaining the observed heart rate responses in our subjects.

The findings of the current study, together with findings from previous similar studies ${ }^{14,15}$ may have implications for cardiovascular training of individuals with SCI. To improve cardiovascular endurance, the training program should be of sufficient frequency, intensity, and duration to induce a stress on the body which is greater than what is regularly encountered during activities of daily living. ${ }^{20}$ Previous research into voluntary exercise training for people with paraplegia has shown that it is beneficial in terms of improving maximal oxygen consumption. ${ }^{21}$ However, it is not clear whether the improvement in maximal oxygen consumption is a result of any 'central' cardiovascular improvement. Therefore, it is reasonable to expect that exercise which promotes 'peripheral' venous return, increases cardiac volume loading ${ }^{15}$ and induces a greater metabolic stress in the absence of an increased heart rate, such as the case with combined arm + ES-induced leg cycling exercise, may lead to greater gains in cardiovascular fitness than voluntary upper body exercise alone.

\section{Acknowledgements}

The authors wish to acknowledge the support of the National Heart Foundation, the National Health and Medical Research Council, and scholarship funds provided by the Faculty of Health Sciences. Our sincerest appreciation is also forwarded to the subjects who volunteered for this project. Lastly, the authors wish to 
acknowledge the motivation and unconditional support in all our endeavours, of Professor John Sutton, who died on February 7, 1996.

\section{References}

1 Hjeltnes N. Oxygen uptake and cardiac output in graded arm exercise in paraplegics with low level spinal lesions. Scand $J$ Rehab Med 1977; 9: 107-113.

2 Kinzer SM, Convertino VA. Role of leg vasculature in the cardiovascular response to arm work in wheelchair-dependent populations. Clin Phys 1989; 9: 525-533.

3 Davis GM. Exercise capacity of individuals with paraplegia. Med Sci Sports Ex 1993; 25: 423-432.

4 Glaser RM. Exercise and locomotion for the spinal cord injured. In Terjung RL (ed.) Exercise and Sport Science Reviews. Vol. 13. Macmillan Publishers, New York, 1985.

5 Mathias CJ, Frankel HL. Cardiovascular control in spinal man. Ann Rev Phys 1988; 50: 577-592.

6 Hopman MTE. Circulatory responses during arm exercise in individuals with paraplegia. Int J Sports Med 1994; 15: 126-131.

7 Davis GM, Shephard RJ. Cardiorespiratory fitness in highlyactive versus inactive paraplegics. Med Sci Sports Ex 1988; 20: $463-468$

8 Hopman MTE, Oeseburg B, Binkhorst RA. Cardiovascular responses in paraplegic subjects during arm exercise. Eur J Appl Phys 1992; 65: $73-78$

9 Davis GM et al. Cardiovascular responses to arm cranking and FNS-induced leg exercise in paraplegics. J Appl Phys 1990; 69: $671-677$.

10 Figoni SF et al. FNS-assisted venous return in exercising SCI men. Proceedings of the International Conference of the Association of Advanced Rehabilitation Technology. Montreal. 1988, pp. $328-329$.
11 Davis GM, Thomas AJ, Sutton JR. Cardiovascular and metabolic responses to electrical stimulation-induced leg exercise. Med Sci Sports Ex 1995; 27: S138.

12 Glaser RM. Arm exercise training for wheelchair users. Med Sci Sports Ex 1989; 21: S149-157.

13 Glaser RM et al. Use of lower-limb FNS to improve arm exercise performance of SCI individuals. Proceedings of the RESNA 16th Annual Conference. pp 416-418, 1993

14 Raymond J, Davis GM, Climstein M, Sutton JR. Cardiorespiratory responses to arm cranking versus arm and electrical stimulation-induced leg cycling in SCI. Proceedings of the International Medical Society of Paraplegia, Sydney, Australia, 1995.

15 Hooker et al. Metabolic and hemodynamic responses to concurrent voluntary arm crank and electrical stimulation leg cycle exercise in quadriplegics. J Rehab Res Dev 1992; 29: 1-11.

16 Goldberger AL, Goldberger N. Clinical Electrocardiography. A Simplified Approach. 3rd ed. The C.V. Mosby Company, St Louis, USA, 1986.

17 Rowell LB. Human Cardiovascular Control. Oxford University Press, New York, 1993.

18 Ashley EA et al. Evidence of autonomic dysreflexia during functional electrical stimulation in individuals with spinal cord injuries. Paraplegia 1993; 31: 593-605.

19 Smith GDP, Watson LP, Pavitt DV, Mathias CJ. Abnormal cardiovascular and catecholamine responses to supine exercise in human subjects with sympathetic dysfunction. J Physiol 1995; 484: $255-265$.

20 Astrand P-O, Rodahl K. Textbook of Work Physiology. Physiological Bases of Exercise 3rd ed. McGraw-Hill Book Company, New York, 1986.

21 Davis GM. Exercise capacity following spinal cord injury. In: Cardiovascular and Respiratory Responses to Exercise in Health and Disease. JR Sutton \& R Balnave (eds). Sydney: University of Sydney. pp 179-192, 1992. 